\title{
AN OPERATIONAL REPRESENTATION OF THE ADDITION THEOREMS FOR SPHERICAL WAVES*
}

\section{By Ari Ben-Menahem}

1. Introduction. In problems of wave propagation, it is sometimes necessary to transform the wave-functions in one coordinate system into another system which is convenient for the boundary-value problem in question. Such a transformation is usually achieved by an "addition theorem" which relates the eigen functions of the two systems. Sato ${ }^{1}$ has obtained an addition theorem for $h_{n}^{(1)}(\kappa R) P_{n}^{m}(\cos \theta) e^{i m \varphi}$ valid for a translation of the origin along the $Z$-axis. Friedman and Russek ${ }^{2}$ obtained addition theorems for standing, converging and diverging spherical waves under conditions of a combined rotation and translation of the spherical coördinate system. It will be shown here that their results can be condensed into a relatively simple form which is advantageous in many applications. For the reader's benefit, we have preserved the notation of Friedman and Russek ${ }^{2}$ with minor changes.

2. The Expansion for $j_{n}\left({ }_{k} R\right) P_{n}^{m}(\cos \theta) e^{i m \varphi}$. Consider a point $P$ which has the spherical coördinates $(R, \theta, \varphi)$ with respect to the origin $O$. A new origin $O^{\prime}$ has the coördinates $\left(r_{0}, \theta_{0}, \varphi_{0}\right)$ with respect to $O$. Let the spherical coördinates of $P$ with respect to $O^{\prime}$ be $\left(r, \theta^{\prime}, \varphi^{\prime}\right)$. Then, the eigen functions $j_{n}(\kappa R) P_{n}^{m}(\cos \theta) e^{i m \varphi}$ with respect to $O$ are expressed in terms of the eigen functions $j_{\nu}(\kappa r) P_{\nu}^{\mu}\left(\cos \theta^{\prime}\right) e^{i \mu \varphi^{\prime}}$ with tespect to $O^{\prime}$, through the expansion ${ }^{2}$ :

$$
\begin{aligned}
& j_{n}(\kappa R) P_{n}^{m}(\cos \theta) e^{i m \varphi} \\
&=\sum_{\nu=0}^{\infty} \sum_{\nu=-\nu}^{\nu} i^{n-\nu}(2 \nu+1) \frac{(\nu-|\mu|) !}{(\nu+|\mu|) !} j_{\nu}(\kappa r) P_{\nu}^{\mu}\left(\cos \theta^{\prime}\right) e^{-i \mu \varphi^{\prime}} \\
& \quad \times\left\{e^{i(m+\mu) \varphi_{0}} \sum_{p} i^{p} a(|m|,|\mu| ; p, n, \nu) j_{p}\left(\kappa r_{0}\right) P_{p}^{m+\mu}\left(\cos \theta_{0}\right)\right\}
\end{aligned}
$$

with

$$
\begin{aligned}
& a(|m|,|\mu| ; p, n, \nu)=\frac{(2 p+1)(n+\nu-p-1) ! !}{(n+p-\nu) ! !(\nu+p-n) ! !(p+\nu+n+1) ! !} \\
& \times \sum_{j=0}^{\rho}\left(\begin{array}{c}
\rho \\
j
\end{array}\right) \frac{(n+j+|m|) !(\nu-|m|-j+p) !}{(n-j-|m|) !(\nu+|m|+j-p) !} e^{\pi i\{\{(n+p-\nu)+|m|+j\}}
\end{aligned}
$$

where $p$ runs over the set $\nu+n, \nu+n-2 \cdots \nu-n, \rho=p-|m|-|\mu|$ and $(s) ! !=s(s-2) \cdots 2$ or $1,(0) ! !=(-1) ! !=1$. Note that the expression inside the curly braces in eq. (1) depend on the coördinates $\left(r_{0}, \theta_{0}, \varphi_{0}\right)$ alone.

* Contribution No. 1074, Division of Geological Sciences California Institute of Technology, Pasadena, California.

1 Y. Sato., Bull. Earth. Res. Inst. Tokyo, 28, 1-22 and 175-217 (1950).

2 B. Friedman and J. Russek, Quart. App. Math. 12, 13-23 (1954). 
We wish now to transform this expression into one in which the coefficients $a(|m|,|\mu| ; p, n, \nu)$ do not appear. We make use of an interesting result given by Erdelyi ${ }^{3}$ :

$$
i^{p} j_{p}\left(\kappa r_{0}\right) P_{p}^{m+\mu}\left(\cos \theta_{0}\right) e^{i(m+\mu) \varphi_{0}}=(-D)^{m+\mu} P_{p}^{(m+\mu)}(\partial / \partial i \kappa z) j_{0}\left(\kappa r_{0}\right)
$$

with

$$
\begin{gathered}
j_{0}\left(\kappa r_{0}\right)=\frac{\sin \kappa r_{0}}{\kappa r_{0}} \\
\frac{\partial}{\partial z}=\cos \theta_{0} \frac{\partial}{\partial r_{0}}-\frac{1}{r_{0}} \sin \theta_{0} \frac{\partial}{\partial \theta_{0}} \\
D=e^{ \pm i \varphi_{0}}\left\{\sin \theta_{0} \frac{\partial}{\partial i \kappa r_{0}}+\frac{\cos \theta_{0}}{i \kappa r_{0}} \frac{\partial}{\partial \theta_{0}} \pm \frac{i}{i \kappa r_{0} \sin \theta_{0}} \frac{\partial}{\partial \varphi_{0}}\right\}
\end{gathered}
$$

The plus sign in eq. (6) corresponds to the case $m+\mu>0$ while the minus sign corresponds to the case $m+\mu<0$. The right side of eq. (3) is the operational interpretation of $P_{p}^{m+\mu}\left(\partial / \partial i_{\kappa} z\right) j_{0}\left(\kappa r_{0}\right) . P_{p}^{(m+\mu)}$ stands for the $(m+\mu)$ th derivative of the Legendre polynomial $P_{p}$ with respect to its argument. Thus $P_{p}^{(m+\mu)}$ is an operational polynomial of the degree $p-|m|-|\mu|$.

Substituting the relation given in eq. (3) into eq. (1) and using the theorem ${ }^{2}$

$$
P_{n}^{m}(x) P_{\nu}^{\mu}(x)=\sum_{p} a(|m|,|\mu| ; p, n, \nu) P_{p}^{m+\mu}(x)
$$

we obtain at once

$$
\begin{aligned}
& j_{n}(\kappa R) P_{n}^{m}(\cos \theta) e^{i m \varphi} \\
& =i^{n} \sum_{\mu, \nu}(2 \nu+1) \frac{(\nu-|\mu|) !}{(\nu+|\mu|) !} j_{\nu}(\kappa r) P_{\nu}^{\mu}\left(\cos \theta^{\prime}\right) e^{-i \mu \varphi^{\prime}} P_{n}^{m}\left\{j_{\nu}\left(k r_{0}\right) P_{\nu}^{\mu}\left(\cos \theta_{0}\right) e^{i \mu \varphi_{0}}\right\}
\end{aligned}
$$

where $P_{n}^{m}$ is the differential operator $P_{n}^{m}(\partial / \partial i \kappa z)=(-D)^{m} P_{n}^{(m)}(\partial / \partial i \kappa z)$. For the special case $m=0, \theta_{0}=0$ one has for example:

$$
\begin{aligned}
n & =0 & j_{0}(\kappa R) & =\sum_{v=0}^{\infty}(2 v+1) j_{\nu}(\kappa r) j_{\nu}\left(\kappa r_{0}\right) P_{\nu}\left(\cos \theta^{\prime}\right) \\
n & =1 & j_{1}(\kappa R) \cos \theta & =\sum_{v=0}^{\infty}(2 \nu+1) j_{\nu}(\kappa r) j_{\nu}^{\prime}\left(k r_{0}\right) P_{\nu}\left(\cos \theta^{\prime}\right)
\end{aligned}
$$

which are the well-known classical expansions.

3. The Expansions for $h_{n}^{(1)}(\kappa R) P_{n}^{m}(\cos \theta) e^{i m \varphi}$ and $h_{n}^{(2)}(\kappa R) P_{n}^{m}(\cos \theta) e^{i m \varphi}$. The addition theorems for the case $r<r_{0}$ are $^{2}$ :

$$
\begin{aligned}
h_{n}^{(1,2)}(\kappa R) P_{n}^{m} & (\cos \theta) e^{i m \varphi} \\
= & \sum_{\nu=0}^{\infty} \sum_{\mu=-\nu}^{\nu} i^{n-1}(2 r+1) \frac{(\nu-|\mu|) !}{(\nu+|\mu|) !} j_{\nu}(\kappa r) P_{\nu}^{\mu}\left(\cos \theta^{\prime}\right) e^{-i \mu \varphi^{\prime}} \\
& \times\left\{e^{i(m+\mu) \varphi_{0}} \sum_{p} i^{p} a(|m|,|\mu| ; p, n, \nu) h_{p}^{(1,2)}\left(\kappa r_{0}\right) P_{p}^{m+\mu}\left(\cos \theta_{0}\right)\right\}
\end{aligned}
$$

3 A. Erdelyi, Physica 4, 107-120 (1937). 
Making use again of eq. (7) and the relations ${ }^{3}$ :

$$
i^{p} h_{p}^{(1,2)}\left(\kappa r_{0}\right) P_{p}^{m+\mu}\left(\cos \theta_{0}\right) e^{i(m+\mu) \varphi_{0}}=(-D)^{m+\mu} P_{p}^{(m+\mu)}(\partial / \partial i \kappa z) h_{0}^{(1,2)}\left(\kappa r_{0}\right)
$$

with

$$
h_{0}^{(1)}\left(\kappa r_{0}\right)=\frac{e^{i \kappa r_{0}}}{i \kappa r_{0}}, \quad h_{0}^{(2)}\left(\kappa r_{0}\right)=\frac{e^{-i \kappa r_{0}}}{-i \kappa r_{0}}
$$

we obtain for $r<r_{0}$

$h_{n}^{(1,2)}\left({ }_{\kappa} R\right) P_{n}^{m}(\cos \theta) e^{i m \varphi}$

$=i^{n} \sum_{\nu, \mu}(2 \nu+1) \frac{(\nu-|\mu|) !}{(\nu+|\mu|) !} j_{\nu}(\kappa r) P_{\nu}^{\mu}\left(\cos \theta^{\prime}\right) e^{-i \mu \varphi^{\prime}} P_{n}^{m}\left\{h_{\nu}^{(1,2)}\left(\kappa r_{0}\right) P_{\nu}^{\mu}\left(\cos \theta_{0}\right) e^{i \mu \varphi_{0}}\right\}$

where $P_{n}^{m}$ has the same meaning as before. Note that equations (8) and (14) have the same form. The special case $m=0, \theta_{0}=0$ yields results similar to equations (9) and (10).

The case $r>r_{0}$ is different; the addition theorems yield ${ }^{2}$ :

$$
\begin{aligned}
h_{n}^{(1,2)}(\kappa R) P_{n}^{m} & (\cos \theta) e^{i m \varphi} \\
= & \sum_{\nu=0}^{\infty} \sum_{\mu=-\nu}^{\nu} i^{n-\nu}(2 \nu+1) \frac{(\nu-|\mu|) !}{(\nu+|\mu|) !} j_{\nu}\left(\kappa r_{0}\right) P_{\nu}^{\mu}\left(\cos \theta^{\prime}\right) e^{-i \mu \varphi^{\prime}} \\
& \times\left\{e^{i(m+\mu) \varphi_{0}} \sum_{p} i^{p} a(|m|,|\mu| ; p, n, \nu) h_{p}^{(1,2)}(\kappa r) P_{p}^{m+\mu}\left(\cos \theta_{0}\right)\right\}
\end{aligned}
$$

The presence of $h_{p}^{(1,2)}(\kappa r)$ inside the curly braces does not permit us to repeat the former procedure for the general case. However, for $m=0, \theta_{0}=0$ we have, similar to the derivation of equations (8) and (14):

$h_{n}^{(1,2)}(\kappa R) P_{n}(\cos \theta)=i^{n} \sum_{\nu=0}^{\infty}(2 \nu+1) j_{\nu}\left(\kappa r_{0}\right) P_{\nu}\left(\cos \theta^{\prime}\right) P_{n}\left(\partial / \partial i_{\kappa} r\right) h_{\nu}^{(1,2)}(\kappa r)$

4. Transformation of the Field-Potentials. Let the potential $\phi(R, \theta, \varphi)$ be expanded into a series of spherical wave functions in the spherical coordinate system $O$.

$$
\phi=\sum_{n=0}^{\infty} \sum_{m=-n}^{n}\left\{A_{m n} j_{n}(\kappa R)+B_{m n} h_{n}^{(1)}(\kappa R)\right\} P_{n}^{m}(\cos \theta) e^{i m \varphi}
$$

Since the physical field is independent of the coördinate system used to express it, we may write for $r<r_{0}$ :

$$
\phi=\sum_{\nu=0}^{\infty} \sum_{\mu \rightarrow-}^{\nu}\left\{\alpha_{\mu \nu} j_{\nu}(\kappa r)+\beta_{\mu \nu} j_{\nu}(\kappa r)\right\} P_{\nu}^{\mu}\left(\cos \theta^{\prime}\right) e^{i \mu \varphi^{\prime}}
$$

where $\phi$ is now being expanded with respect to the spherical coördinate system $O^{\prime}$. Using the addition theorems given above, together with the orthogonality properties of the Legendre polynomials, it is not difficult to verify that for the case $r<r_{0}$,

$\left\{\begin{array}{l}\alpha_{\mu \nu} \\ \beta_{\mu \nu}\end{array}\right\}=(2 \nu+1) \frac{(\nu-|\mu|) !}{(\nu+|\mu|) !} \sum_{s=0}^{\infty} \sum_{q=-s}^{s} i^{s}\left\{\begin{array}{l}A_{s q} \\ B_{s q}\end{array}\right\} P_{\varepsilon}^{q}\left(\frac{\partial}{\partial i_{\kappa} z}\right)\left\{\begin{array}{c}j_{\nu}\left(\kappa r_{0}\right) \\ h_{\nu}^{(1)}\left(\kappa r_{0}\right)\end{array} P_{\nu}^{\mu}\left(\cos \theta_{0}\right) e^{i \mu \varphi_{0}}\right\}$

with $P_{s}^{q}=(-D)^{q} P_{s}^{(q)}$ as before. 
For the case $r>r_{0}$ and $m=0, \theta_{0}=0$, we represent $\phi$ in the form:

$$
\phi=\sum_{\nu=0}^{\infty}\left\{\alpha_{\nu} j_{\nu}(\kappa r)+\beta_{\nu}(r) j_{\nu}\left(\kappa r_{0}\right)\right\} P_{\nu}\left(\cos \theta^{\prime}\right)
$$

where the coefficients $\alpha_{\nu}$ and $\beta_{\nu}(r)$ are found to be:

$$
\begin{aligned}
& \alpha_{\nu}=(2 \nu+1) \sum_{s=0}^{\infty} i^{8} A_{s} P_{s}\left(\partial / \partial i \kappa r_{0}\right) j_{\nu}\left(\kappa r_{0}\right) \\
& \beta_{\nu}=(2 \nu+1) \sum_{s=0}^{\infty} i^{s} B_{s} P_{s}(\partial / \partial i \kappa r) h_{\nu}^{(1)}(\kappa r)
\end{aligned}
$$

Comparing the original addition theorems as given in equations (1), (11) and (15) to their operational representation as given in equations (8), (14) and (16), we may conclude that while the original theorems are better fitted for numerical computations, the operational representation facilitates algebraic as well as analytic manipulations with the field expansions. To this extent the two representations are supplementary.

(Received February 27, 1962) 